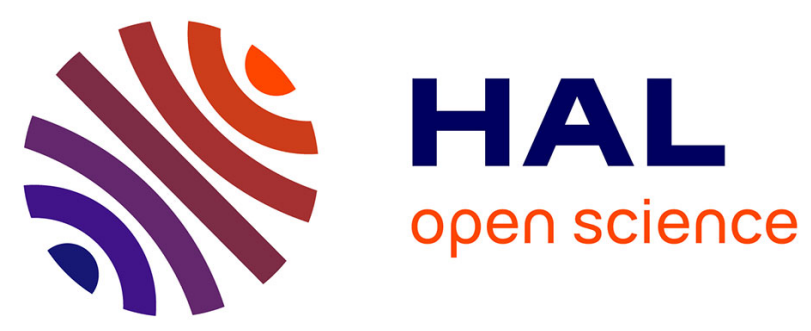

\title{
Short-Term Impact of tDCS Over the Right Inferior Frontal Cortex on Impulsive Responses in a Go/No-go Task
}

Salvatore Campanella, Elisa Schroder, Marie-Anne Vanderhasselt, Chris Baeken, Charles Kornreich, Paul Verbanck, Boris Burle

\section{To cite this version:}

Salvatore Campanella, Elisa Schroder, Marie-Anne Vanderhasselt, Chris Baeken, Charles Kornreich, et al.. Short-Term Impact of tDCS Over the Right Inferior Frontal Cortex on Impulsive Responses in a Go/No-go Task. Clinical EEG and Neuroscience, 2018, 10.1177/1550059418777404 . hal-01803847

\section{HAL Id: hal-01803847 https://hal.science/hal-01803847}

Submitted on 30 Apr 2019

HAL is a multi-disciplinary open access archive for the deposit and dissemination of scientific research documents, whether they are published or not. The documents may come from teaching and research institutions in France or abroad, or from public or private research centers.
L'archive ouverte pluridisciplinaire HAL, est destinée au dépôt et à la diffusion de documents scientifiques de niveau recherche, publiés ou non, émanant des établissements d'enseignement et de recherche français ou étrangers, des laboratoires publics ou privés. 
See discussions, stats, and author profiles for this publication at: https://www.researchgate.net/publication/325315781

\section{Short-Term Impact of tDCS Over the Right Inferior Frontal Cortex on Impulsive Responses in a Go/No-go Task}

Article in Clinical EEG and neuroscience: official journal of the EEG and Clinical Neuroscience Society (ENCS) · May 2018

DOI: $10.1177 / 1550059418777404$

CITATIONS

2

7 authors, including:

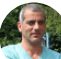

Salvatore Campanella

Université Libre de Bruxelles

123 PUBLICATIONS 3,467 CITATIONS

SEE PROFILE

Marie-Anne Vanderhasselt

Ghent University

89 PUBLICATIONS 1,974 CITATIONS

SEE PROFILE
READS

201

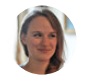

Elisa Schroder

Université Libre de Bruxelles

12 PUBLICATIONS 40 CITATIONS

SEE PROFILE

Chris Baeken

Ghent University

232 PUBLICATIONS 2,894 CITATIONS

SEE PROFILE

Some of the authors of this publication are also working on these related projects:

PrevenD: Optimization and implementation of cognitive control training to prevent recurrent depression View project

3rd european conference on Brain stimulation in psychiatry - Lyon 18-20th October 2018 View project 


\title{
Short-Term Impact of tDCS Over the Right Inferior Frontal Cortex on Impulsive Responses in a Go/No-go Task
}

Clinical EEG and Neuroscience

I-9

(C) EEG and Clinical Neuroscience Society (ECNS) 2018

Reprints and permissions: sagepub.com/journalsPermissions.nav DOI: 10.1 I77/I5500594/8777404 journals.sagepub.com/home/eeg (SSAGE

\author{
Salvatore Campanella', Elisa Schroder', Marie-Anne Vanderhasselt ${ }^{2,3,4}$, \\ Chris Baeken ${ }^{2,3}$, Charles Kornreich', Paul Verbanck', and Boris Burle ${ }^{5}$
}

\begin{abstract}
Inhibitory control, a process deeply studied in laboratory settings, refers to the ability to inhibit an action once it has been initiated. A common way to process data in such tasks is to take the mean response time (RT) and error rate per participant. However, such an analysis ignores the strong dependency between spontaneous RT variations and error rate. Conditional accuracy function (CAF) is of particular interest, as by plotting the probability of a response to be correct as a function of its latency, it provides a means for studying the strength of impulsive responses associated with a higher frequency of fast response errors. This procedure was applied to a recent set of data in which the right inferior frontal gyrus (rIFG) was modulated using transcranial direct current stimulation $(t D C S)$. Healthy participants $(n=40)$ were presented with a "Go/No-go" task (click on letter $M$, not on letter $W$, session I). Then, one subgroup $(n=20)$ was randomly assigned to one 20-minutes neuromodulation session with tDCS (anodal electrode, rIFG; cathodal electrode, neck); and the other group $(n=20)$ to a condition with sham (placebo) tDCS. All participants were finally confronted to the same "Go/No-go" task (session 2). The rate of commission errors (click on W) and speed of response to Go trials were similar between sessions I and 2 in both neuromodulation groups. However, CAF showed that active tDCS over rIFG leads to a reduction of the drop in accuracy for fast responses (suggesting less impulsivity and greater inhibitory efficiency), this effect being only visible for the first experimental block following tDCS stimulation. Overall, the present data indicate that boosting the rIFG may be useful to enhance inhibitory skills, but that CAF could be of the greatest relevance to monitor the temporal dynamics of the neuromodulation effect.
\end{abstract}

\section{Keywords}

tDCS, inhibition, right inferior frontal gyrus, impulsive errors, conditional accuracy function (CAF)

Received November 9, 20I7; revised April 17, 20I8; accepted April 2I, 2018.

\section{Introduction}

The ability to stop the execution of an automatic reaction or a planned motor behavior is called "response inhibition." Such a concept refers to different aspects of action inhibition such as "action restraint," that is, when a not-yet-initiated action has to be restrained, and "action cancellation," that is, when already initiated responses that are no longer required or become inappropriate in ever-changing environments, have to be cancelled. ${ }^{2}$ Both mechanisms rely on a common network of neural activation, including bilateral inferior frontal regions, the (pre)supplementary motor area (SMA), and thalamic regions, even if additional neural components that are distinct to action restraint (ie, the right superior frontal gyrus and anterior cingulate cortex) or action cancellation (ie, the right middle frontal gyrus and the posterior cingulate cortex) have also been described. ${ }^{3}$ Action restraint has been deeply studied in laboratory settings by using the "Go/No-go" task. In this task, participants must press a button in response to one type of frequently presented stimuli ("Go") and withhold that response to some rare and random stimuli of another type ("No-go"). ${ }^{4}$ In other words, a prepotent motor response is installed (click to frequent Go trials), but participants have to withhold this dominant response when a No-go trial appears. This process is

'Laboratoire de Psychologie Médicale et d'Addictologie, ULB Neuroscience Institute (UNI), CHU Brugmann-Université Libre de Bruxelles (U.L.B.), Brussels, Belgium

${ }^{2}$ Department of Psychiatry and Medical Psychology, Ghent University, Ghent, Belgium

${ }^{3}$ Department of Psychiatry, University Hospital (UZBrussel), Brussels, Belgium

${ }^{4}$ Department of Experimental Clinical and Health Psychology, Ghent University, Belgium

${ }^{5}$ Aix-Marseille Universite', Centre National de la Recherche Scientifique, LNC Unite' Mixte de Recherche, Marseille, France

Corresponding Author:

Salvatore Campanella, The Belgian Fund for Scientific Research (F.N.R.S.), CHU Brugmann, Psychiatry Secretary, 4, Place Vangehuchten, B-I020

Brussels, Belgium.

Email: salvatore.campanella@chu-brugmann.be 
seen as a key component of executive control, affecting an individual's ability to inhibit impulsive responses to stimuli. When impaired, it has therefore been widely related to many behaviors requiring impulse suppression such as food consumption, smoking urges, or alcohol-seeking behavior. ${ }^{5}$ In this view, a lot of studies tried to verify whether this inhibitory process can be rehabilitated in order to improve an individual's ability to overrule impulsive reactions and regulate behavior in a consistent way with long-term goals. A recent meta-analysis showed that a small but homogeneous effect of "cognitive training" on behavior was found $(d+=0.378,95 \%$ $\mathrm{CI}=[0.258,0.498]){ }^{6}$ This suggests that inhibitory control training may be a useful intervention technique for reducing health risk behaviors. ${ }^{6}$

Similarly, transcranial direct current stimulation (tDCS), a noninvasive brain stimulation technique, is based on the application of weak (0.5-2 mA), direct electric signals into the brain through relatively large electrodes placed over the scalp. tDCS devices essentially comprise 4 main components: electrodes (1 anode and 1 cathode), a power supply ( $9 \mathrm{~V}$ battery), an ampere meter (to measure the intensity of the electric current), and a potentiometer (to allow adjustment of the electric current). This technique has been successfully used as a tool to improve behavioral inhibition. ${ }^{7,8}$ tDCS of the dorsolateral prefrontal cortex (DLPFC) has already been used in many addictive disorders: It has been shown to decrease craving among crack cocaine, ${ }^{9}$ marijuana, ${ }^{10}$ appetitive food,${ }^{11}$ tobacco, ${ }^{12}$ and alcohol users. ${ }^{13}$ Overall, a recent meta-analysis has found 17 studies dealing with the effects of neurostimulation on substances and appetitive foods. ${ }^{14}$ In the active DLPFC stimulation, a decrease of craving compared to sham stimulation is reported, with an effect size of 0.476 , providing clear evidence that noninvasive neurostimulation of the DLPFC decreases craving levels in substance dependence. As a consequence, a level B recommendation (probable efficacy) is conferred for the clinical effect of anodal tDCS of the right DLPFC with the cathode over the left DLPFC in addiction/craving, although this statement covers different clinical conditions and outcome variables. ${ }^{15}$ However, the available literature is still relatively small and sparse, and the long-term safety and clinical efficacy of these interventions need to be confirmed. ${ }^{16}$ Indeed, "recommending" a type of tDCS protocol in a given clinical situation according to the level of evidence of its "real" efficacy (comparing active vs sham condition) may be statistically relevant but not clinically meaningful in daily living or in terms of quality of life. For instance, while Klauss et $\mathrm{al}^{17}$ showed that tDCS on bilateral DLPFC did not diminish craving but reduced relapse probability in 33 alcohol dependent patients, da Silva et al ${ }^{18}$ reported a suppressed craving but an unexpected trend for more relapse in the active DLPFC group than in the sham group. Therefore, it remains to be determined how to optimize tDCS protocols and techniques to give them "therapeutic relevance" in routine clinical practice. Multiple factors are likely to contribute to the variability of data in tDCS studies ${ }^{19}$ : Among these, a main factor concerns the relevance of the site of stimulation with regard to the tagged cognitive function. ${ }^{20}$ In this view, tDCS over the right inferior frontal gyrus (rIFG) has been shown to boost inhibitory control in a wide range of circumstances. Several studies have shown that unilateral anodal tDCS over the right inferior frontal cortex (rIFC) improves the ability to inhibit responses in healthy subjects using the stop signal task, ${ }^{21-23}$ and modulated its electrophysiological correlate ${ }^{24}$ as the facilitated response inhibition induced by tDCS activation is linked to decreased theta band activity (compared with the sham condition). Similarly, some studies also disclosed some indirect effects of improving inhibition skills through rIFG stimulation, as decreasing anger ruminations, ${ }^{25}$ improving successful lying, ${ }^{26}$ or even improving speech-motor output in recovering patients from poststroke aphasia. ${ }^{27}$ Another theoretical dimension may also explain the heterogeneity of the results. Indeed, inhibition is nowadays not seen any more as a unitary construct, and, the dual mechanisms of control mode ${ }^{28}$ offered to divide cognitive control in 2 different kind of strategies: proactive cognitive control, mainly relying on the active maintenance of contextual information relevant to the ongoing task, and reactive cognitive control, a form of transient control triggered by an external cue. More recent studies therefore suggest that tDCS activation over the rIFG facilitated both pro- and reactive inhibitory strategies in a stop-signal task ${ }^{29}$ while bilateral stimulation of the IFG seems to specifically enhance proactive inhibition (the behavioral reactive responses remaining unaffected in combined stop-signal and Go/No-go tasks). ${ }^{30}$ Of course, here again some mixed results have been reported, as for instance, den Uyl et $\mathrm{al}^{31}$ did not report any impact of rIFG activation (compared with sham) on craving for heavy drinkers, while Dambacher et $a^{32}$ reported no impact of rIFG tDCS activation on the ability to inhibit motor responses engaged in aggressive behaviors. Further studies have then still to clarify the specific improvements that may be triggered by rIFG activation as well as their precise clinical applicability.

A recent study of Campanella et $\mathrm{a}^{33}$ tested the impact of tDCS over the right frontal inferior gyrus (rIFG) when healthy participants are asked to achieve a "Go/No-go" task. Participants received either active tDCS stimulation of the rIFG (tDCS group), or placebo sham neuromodulation (Sham group) over the same region. "Go/No-go" task was assessed before and after the neuromodulation. Because inhibition is a covert process that, when successful, produces little or no overt, measurable behavior, ${ }^{34}$ the authors simultaneously recorded event-related potentials to gain understanding of these hidden processes. The main reported result concerned the decreased P3d amplitude recorded in session 2 (after tDCS application) compared with session 1 (before tDCS application), only in the tDCS group. Increased No-go P3 amplitudes are commonly interpreted as indicators that increased cognitive resources are being recruited for inhibition. ${ }^{35,36}$ The lower P3d amplitudes in tDCS participants may hence attest to the need for less recruitment of neural resources required to correctly perform the task: People confronted with tDCS over the rIFG needed less neural resources to perform the Go/No-go task during session 2, indexing that fewer cortical resources are required to achieve correct inhibitions after 
a single session of 20 minutes. However, the authors did not observe any global increase in performance accuracy between the 2 sessions. This contrasts with some previous studies that have already shown that unilateral anodal tDCS over the rIFG improved the ability to inhibit responses in healthy subjects using a stop signal task. ${ }^{21-23}$ A possible explanation could be that the global false alarm rate might not be sensitive enough to detect subtle changes in impulse control.

Indeed, when confronted to a "Go/No-go" task, classical behavioral analyses, indexing inhibitory control efficiency, concerned (1) the number of errors a subject makes on No-go trials, that is, "false alarms" (or commissions errors) referring to the number of hits when they should not; (2) the number of "omissions," that is, when participants do not click on Go trials; and (3) speed of response (response time [RTs]) to Go trials, as time pressure is known to generate a higher rate of false alarms on No-go trials. ${ }^{37} \mathrm{~A}$ common way to process data in such a task is to take the mean RT and error rate per participant. However, such an analysis ignores the strong dependency between spontaneous RT variations and error rates: it is well established that RTs vary spontaneously, and that when RTs are short, the likelihood of an error increases. This drop in accuracy indexes the tendency to react prematurely based on insufficient accumulated information, which is the core of impulsive behavior. ${ }^{5}$

In this view, "conditional accuracy function" (CAF) is of particular interest, as by plotting the probability of a response to be correct as a function of its latency, it provides a means for studying the strength of impulsive (ie, fast) responses associated with a higher frequency of fast response errors. ${ }^{38} \mathrm{~A}$ welldocumented finding is that in conflict tasks (such as the Stroop task, the Simon task, or the Eriksen flankers tasks), for a relatively large proportion of the fast responses, action selection is captured by automatically activated extraneous stimulusresponse associations to such an extent that deliberate intention-driven action selection is bypassed and an overt response error is committed. ${ }^{39}$ In the Stroop task, for example, a drop in accuracy is classically observed for fast incongruent trials, revealing a tendency for impulsive response to the word name, although irrelevant for the task at hand. ${ }^{38}$ In the situation of a Go/No-go task, it has also been shown that the instruction to respond fast affects the speed of response activation and the strength of response inhibition, as the error rate decreased on longer response latencies. ${ }^{40}$ In other words, when responses are faster, they are more often false alarms (ie, commission errors), reflecting an impulsive response tendency. The depth of this drop in response accuracy to fast responses therefore provide a more sensitive measure of impulse strength as compared with a general/global accuracy rate. ${ }^{38,41}$

With this in mind, if tDCS improves impulse control, its effect should be especially apparent for fast responses, increasing accuracy for those trials, and this might have been masked by global accuracy measures. The main objective of the present article is to reanalyze the set of behavioral data obtained in the study by Campanella et $\mathrm{al}^{33}$ with CAF. This would help us assess changes in accuracy present in the fast responses, hidden in the global (average) analysis of accuracy. Our main hypothesis is that, if tDCS over the rIFG increases inhibitory efficiency over impulsive response (in session 2 compared with session 1), we expect the drop in accuracy for fast response to be reduced after the active tDCS session.

\section{Method}

Full details concerning method can be found in the article by Campanella et al. ${ }^{33}$ In the present study, only data useful for the current behavioral reanalysis are reported.

\section{Participants}

Forty males students (20-30 years old) were enrolled at the University of Ghent. These individuals had normal or corrected vision, and normal hearing. They were not taking any medication and had no history of neurological or psychiatric disease, as confirmed by the English version of the MINI Screen 5.0.0. ${ }^{42}$ Individual scores were obtained for the following questionnaires: the Alcohol Use Disorder Identification Test (AUDIT; Dutch version ${ }^{43}$ ); the Barratt Impulsiveness Scale (BIS-11; Dutch version ${ }^{44}$ ); the State-Trait Anxiety Inventory (STAIT; Dutch version ${ }^{45}$ ); and the Beck Depression Inventory (BDI-II; Dutch version ${ }^{46}$ ). Among these participants, one was found to have reaction times in response to Go trials that were too slow (3 SDs above the mean), and 4 others made too many commission errors (3 SDs above the mean) in the Go/No-go task. Therefore, 35 participants were included in the final sample used for statistical analyses, with 18 in the tDCS group and 17 in the Sham group. The complete characteristics of the cohort are reported in Table 1. The ethics committee of the University of Ghent approved the study, and informed written consent was obtained from each participant. They were paid $€ 25$ for their full participation.

\section{Tasks and Procedure}

When they arrived at the laboratory, participants were confronted with the Go/No-go task. They had to press a button with the index finger of their right hand, as quickly and accurately as possible, whenever the letter M (Go) was displayed, and to withhold pressing the button when the letter W (No-go) was displayed. Both letters were superimposed on a black background. The stimuli consisted of 2 yellow capital letters $(\mathrm{M}$ and $\mathrm{W}$; size $500 \times 400 \mathrm{~mm}$ ) in Arial font displayed on a 17-inch monitor. Participants were placed $1 \mathrm{~m}$ from the screen. Overall, the task comprised 2 separate stimulation blocks lasting around 3 minutes each. Each block contained 133 letters, divided into 93 Go (70\%) and 40 No-go (30\%) letters. Go and No-go letters were displayed in a semirandom order to avoid the consecutive presentation of 2 No-go letters within each block. One to 4 Go letters could precede No-go letters. Each block consisted of the presentation of a black background screen $(500 \mathrm{~ms})$, then the letter $\mathrm{M}$ or $\mathrm{W}$ appeared on this background screen for $200 \mathrm{~ms}$, followed by a return to the initial 
Table I. Characteristics of Transcranial Direct Current Stimulation (tDCS) and Sham Participants Presented as Means (Standard Deviations).

\begin{tabular}{lcrr}
\hline & tDCS $(\mathrm{n}=18)$ & Sham $(\mathrm{n}=17)$ & $P^{\mathrm{a}}$ (Independent $\left.t\right)$ \\
\hline Age (years) & $22.2(3.0)$ & $21.3(1.7)$ & 0.310 \\
Depression (BDI-II score) & $4.7(3.9)$ & $6.0(5.5)$ & $0.4 I I$ \\
Anxiety Trait (STAI- B) & $34.3(8.4)$ & $33.2(6.9)$ & 0.674 \\
Alcohol problems (AUDIT) & $9.7(4.3)$ & $7.5(3.8)$ & 0.126 \\
Impulsivity (BIS-II): & $13.3(3.5)$ & $14.1(3.3)$ & 0.505 \\
BIS-Attentional & $12.9(4.1)$ & $13.6(4.4)$ & 0.628 \\
BIS-Motor Planning & $30.6(4.1)$ & $28.9(6.2)$ & 0.367 \\
BIS-Non Planning & & \\
\hline
\end{tabular}

Abbreviations: BDI, Beck Depression Inventory; STAI B, State and Trait Anxiety Inventory; AUDIT, Alcohol Use Disorders Identification Test; BIS-I I, Barratt Impulsiveness Scale.

${ }^{a}$ All values are $P>.05$ (nonsignificant).

background screen $(1300 \mathrm{~ms})$. Thus, subjects had a maximum of $1500 \mathrm{~ms}$ to press the button before the next letter appeared. Before starting the session, participants had a practice session (20 trials including 3 No-go letters) in order to be sure that instructions were correctly understood. Once they had finished the first session, participants were then randomly assigned to a stimulation condition, active or sham, using a double-blind counterbalanced procedure. Randomization was conducted centrally by an independent researcher using 1:1 randomization with atmospheric (white noise) generated random sequences of integers (without duplicates) as offered by NeuroConn (codes that are associated with real or sham stimulation). This way, a double blind stimulation could be performed. tDCS was applied using a pair of surface sponge rubber electrodes $\left(25 \mathrm{~cm}^{2}\right)$ soaked in saline and was administered by a DC-stimulator (Neuroconn, Ilmenau, Germany). One electrode was placed over the scalp, above the cortical area aimed to be stimulated. The other electrode, the reference, can be positioned elsewhere, for example, over the extracephalic region. In our study, we placed this reference electrode at the left superior region of the trapezius muscle near the base of the participant's neck. The anodal electrode was placed over the rIFG, which corresponds to the F8 electrode. ${ }^{24}$ Participants were then confronted with an active or a sham tDCS session lasting 20 minutes, during which they filled in questionnaires. During sham stimulation, the tDCS ramps up during a period of 30 seconds just as in the active tDCS condition, but is immediately followed by a fade out of 30 seconds. This generates an active placebo condition and makes it difficult for participants to discern between sham and active stimulation.

After the tDCS session, all participants were then confronted with the same Go/No-go task described in session 1.

\section{Data Processing}

All RTs inferior to $100 \mathrm{~ms}$ were considered as anticipations and were hence removed (percentage of rejection: 2.22\%). From the remaining data, CAF were computed as follows. First RT, including correct and false alarm responses, were "vincentized" ${ }^{47}$ " separately for each session and block: Basically, RTs were sorted in ascending order within each block and binned in classes containing the same number of trials (not necessarily a round value), termed "quantiles." In the present analysis, data were binned in 5 quantiles. For each quantile, the mean RT value of the class and the proportion of correct response were computed. The CAF plots the proportion of correct response as a function of mean RT across all quantiles. CAF were computed for each participant and session separately and then, for illustrative purposes, averaged across participants for each condition. Statistical analyses were performed by analyses of variance, with proportion correct as the dependent variable, tDCS conditions (active vs sham) as a between participant factor, and session (pre $=$ session 1 vs post $=$ session2), quantiles and block number (first or second) as within participant factors. When necessary, Greenhouse-Geisser correction for violation of sphericity assumption was applied.

\section{Results}

The CAF for each experimental conditions are presented on Figure 1. First, the overall effect of quantiles was significant, $F(4,132)=25.80, \varepsilon=0.64, P<.001$, indicating that the rate of false alarms increases for fast responses. A general effect of session (pre vs post) also proved significant, $F(1,33)=5.36, P=$ .03 , likely indexing a learning effect. This is further supported by an interaction between session and quantile, $F(4,132)=5.01, \varepsilon$ $=.72, P<.001$. No main effect of stimulation was observed, $F(1,33)=0.13$, but it was qualified by a third-order interaction between stimulation group, session, block number, and quantiles, $F(4,132)=3.62, \varepsilon=0.82, P=.01$. To clarify this interaction, the 2 participants groups (tDCS vs sham) were separately analyzed.

On one hand, for the tDCS group (top row of Figure 1), one observed an effect of quantile, $F(4,68)=18.60, \varepsilon=0.53, P<$ .001 . No main effect of session was observed, $F(1,17)=2.08$, $P=.17$, but session was qualified by several interactions. First, session marginally interacts with quantile, $F(4,68)=2.79, \varepsilon=$ $0.63, P=.06$. It also marginally interacts with block number, $F(1,17)=3.56, P=.08$. More important, the second-order interaction between session, block number, and quantile proved significant, $F(4,68)=3.46 ; \varepsilon=0.74, P=.02$. Based on 

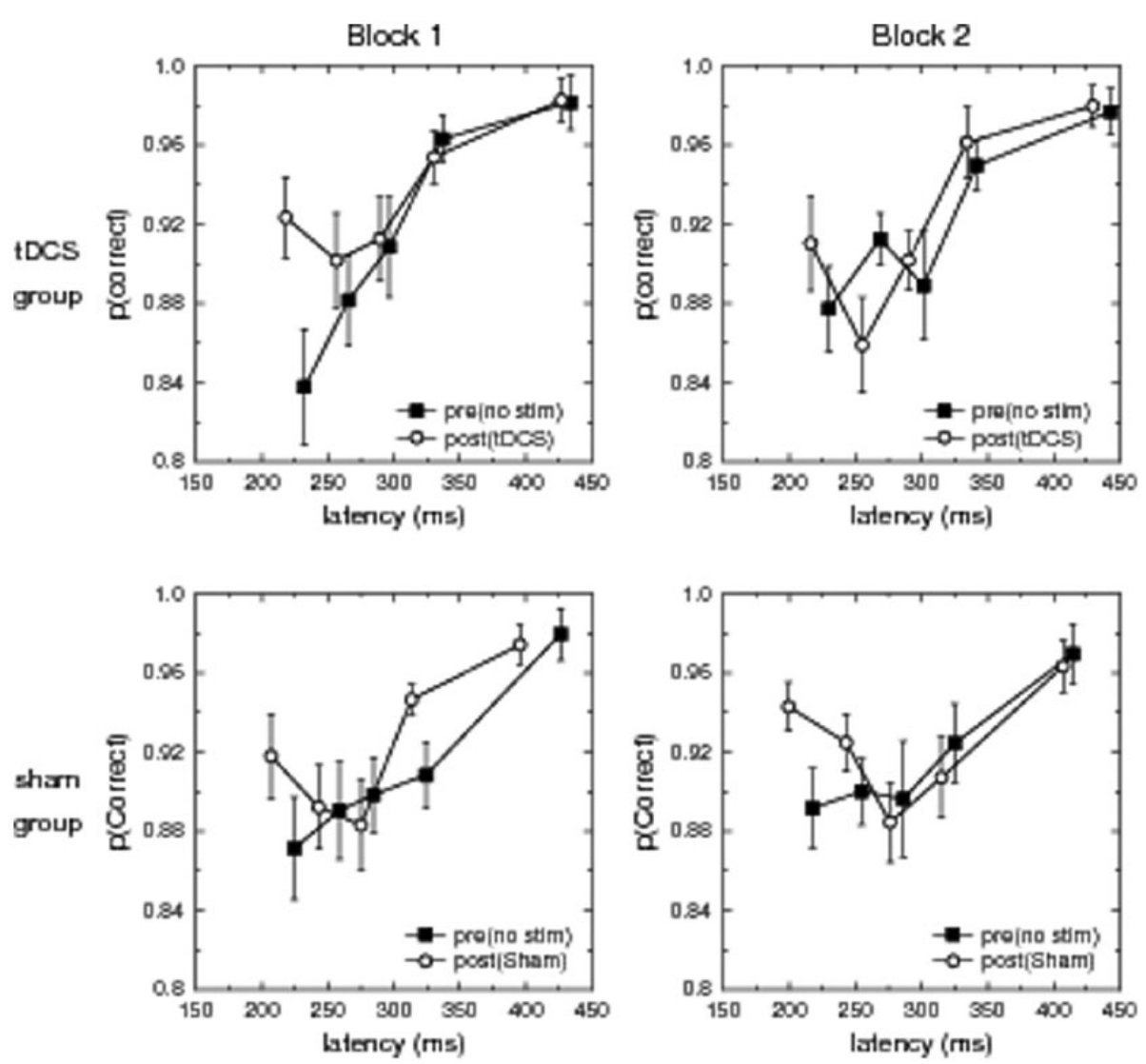

Figure I. Conditional accuracy functions (CAF) for the transcranial direct current stimulation (tDCS) group (top row) and the sham group (bottom row) and for the first (first column) and second (second column) block of each session. CAFs plot the probability that a response is correct as a function of its latency. Errors bars represent the standard error of the mean, computed following the normalization of the variance proposed by Cousineau ${ }^{48}$ (and corrected by Morey ${ }^{49}$ ) for within-participants factors.

inspection of Figure 1 (top row), this interaction may reflect a short lasting effect of the tDCS, being effective on the first block, but with a decreased efficiency for the second block. Separate analysis for the 2 blocks confirmed this: For the first block, an effect of quantile was observed, $F(4,68)=10.47, \varepsilon=$ $0.65, P<.001$, along with an effect of session: without vs with tDCS, $F(1,17)=4.67, P=.05$. Importantly, these 2 factors interact, $F(4,68)=3.52, \varepsilon=0.65, P=.03$, indicating that the shape of the CAF changes between no stimulation (pre) and with stimulation (post). For the second block, the effect of quantile was still significant, $F(4,68)=13.18, \mathcal{E}=0.79, P<.001$, but no effect of session was observed, $F(1,17)<1$. It was, however, qualified by a marginally significant interaction with quantile, $F(4,68)=2.48, \varepsilon=0.72, P=.07$.

On the other hand, for the Sham group (bottom row of Figure 1), one can observe that, as for the tDCS group, the effect of quantile was significant, $F(4,64)=9.59, \varepsilon=0.69, P$ $<.001$. The interaction between quantile and session was just above significance, $F(4,64)=3.23, \mathcal{E}=0.52, P=.05$. However, in contrast with the tDCS group, the interaction between session, block number, and quantile was not significant, $F(4,64)$ $=1.21, \mathcal{E}=0.77, P=.32$. To asses that the interaction between quantile and session for the first blocks of the tDCS group is really specific, we nonetheless analyzed the 2 blocks separately. Although an effect of quantile was present for both blocks in the sham group, $F(4,64)=8.23, \varepsilon=0.63, P<.001$ and $F(4,64)=4.68, \varepsilon=0.77, P=.01$, for blocks 1 and 2 respectively, neither the effect of session, $F(1,16)=2.36, P=$ .14 and $F(1,16)=0.99, P=.33$, for blocks 1 and 2 , respectively, nor the interaction, $F(4,64)=2.06, \varepsilon=0.6, P=.13$ and $F(4,64)=2.39, \varepsilon=0.77, P=.08$, for blocks 1 and 2 , respectively, reached significance. Hence, only the tDCS on the first block did affect the shape of the CAF between session (without vs with stimulation).

In order to point to the necessity of using CAF, classical analyses were also performed on mean individual values for RTs (to Go trials) and for commission errors (see Table 2), using a $2 \times 2 \times 2$ analysis of variance with "Session" (session 1; session 2), Block (block 1; block 2) as the within-subject factors and "Group" (tDCS; sham) as the between-subject factor. While a main Session effect was found both for RTs, $F(1$, $33)=16.044, P<.001$, and errors, $F(1,33)=8.085, P=.008$, no significant interactions (group $\times$ session, group $\times$ block, group $\times$ block $\times$ session) were observable (all $P$ s $\geq .340$ ). 
Table 2. The Go/No-go Task: RTs to Go Stimuli and Number of Errors in tDCS and Sham Participants for Sessions I and 2.

\begin{tabular}{|c|c|c|c|}
\hline & tDCS, Mean (SD) & Sham, Mean (SD) & Mean (SD) \\
\hline Go RTs (ms)-Session I-Block 2 & $313(45)$ & $297(53)$ & $305(49)$ \\
\hline Go RTs (ms)-Session 2-Block 2 & $297(37)$ & $281(37)$ & $289(37)$ \\
\hline Commission error rates (40 trials) Session I-Block I & $8.78(5.7)$ & $9.41(4.8)$ & $9.09(5.2)$ \\
\hline Commission error rates (40 trials) Session I-Block 2 & $8(4.6)$ & $8.76(5.6)$ & $8.37(5)$ \\
\hline Commission error rates (40 trials) Session 2-Block 2 & $7.67(4)$ & $7.47(4.3)$ & $7.57(4.1)$ \\
\hline
\end{tabular}

Abbreviations: RT, response time; tDCS, transcranial direct current stimulation.

\section{Discussion}

Response inhibition has been widely investigated in experimental conditions, for instance, using the "Go/No-go" task. ${ }^{50}$ Campanella and colleagues ${ }^{33}$ showed that people confronted with tDCS over the rIFG needed less neural resources to perform the Go/No-go task during session 2 (after stimulation) than in session 1 (before stimulation), indexing that fewer cortical resources are required to achieve correct inhibition responses after brain stimulation (despite the absence of behavioral accuracy enhancement). At the methodological level, it is important to note that these event-related potential data were grand-averaged data, defined by the average of all correct trials obtained in the 2 blocks of stimulations presented to each participant in each session. At the behavioral level, a common way to process data in such a task is to take the mean RT and error rate per participant. However, the starting point of the present article is that several critiques have suggested that such processes might be more accurately depicted if current measures, often relying on averages across time, were replaced with more temporally sensitive techniques able to depict dynamic change. ${ }^{51,52}$ While "static" measures would not allow deciphering the temporal dynamics of inhibitory responses, distribution analysis will do it. Indeed, an efficient inhibitory response to a No-go trial requires a good balance of speed and accuracy, which is obtained by controlling the relative contribution of response activation and inhibition. ${ }^{53}$ Bias toward activation can cause impulsive behavior, whereas bias toward inhibition can lengthen response times (RT) to urgent events. ${ }^{40}$ In such an activation-suppression model, ${ }^{54}$ the susceptibility to react impulsively is revealed by the relation between fast errors and response speed: stronger initial impulsivity on No-go trials is expressed by an increase in the proportion of fast errors as less time is available for the build-up of suppression to counter this incorrect activation. ${ }^{55}$

With this in mind, CAF provides a means for studying the strength of impulsive responses associated with a higher frequency of fast response errors. Indeed, responding fast (to Go trials) increases the risk of false alarms (ie, responding to No-go stimuli), reflecting an impulsive response tendency. By applying CAF to the current set of data, we could show that, as expected, active tDCS over rIFG leads to a reduction of the drop in accuracy for fast responses (suggesting therefore less impulsivity and then greater inhibitory efficiency). rIFG is core node of the inhibitory network along with (pre)supplementary motor area (SMA) and basal ganglia (especially the subthalamic nucleus, STN). ${ }^{56}$ Recently, CAF analysis in a conflict task during, which tDCS was applied over (pre)SMA revealed a similar reduction of impulsive responses. ${ }^{57}$ However, by comparing overt (behavioral) and covert (EMG) impulsive tendencies, the authors could show that tDCS over (pre)SMA does not modulate the strength of prepotent response activation, but selectively improved the capacity to overcome initial impulsive response activation. Whether rIFG also acts in the same way, or instead reduces the impulsive tendency remain to be deciphered.

A second main result of the present article is that the reported effect was present only for the first experimental block following tDCS stimulation. This suggests an acute effect of the 20 minutes tDCS stimulation. Such a result highlights the absolute necessity to better define the number of sessions that is needed to impact a cognitive function in the long-term. For this purpose, CAF could reveal to be of the highest relevance, as it can furnish an easy way (simple analysis on behavioral data) to monitor when the tDCS effect vanished. If tDCS has been successfully used as a tool to improve diverse cognitive skills in substance use disorder, ${ }^{19}$ results from randomized controlled trials and meta-analyses are mixed, ${ }^{58}$ mainly due to the heterogeneity of the tDCS administration protocols of these studies. CAF could be considered in further studies as an easy method to favor a better knowledge of the number of sessions needed to reach long-term efficiency.

Obviously, this article suffers from some limitations. The main one was related to the fact that, unfortunately, we have to limit our analyses in the present article to the behavioral data. Indeed, it would have been nice to show that the reduction of the P3d amplitude observable for the active tDCs group in session 2 was also "only" present for block 1 . However, the limited number of No-go trials available per block (40 trials minus errors minus artifacted trials) implies that this analysis is impossible due to a poor signal-to-noise ratio. Further studies should increase number of trials by block in order to check whether the observed acute behavioral effect (revealed by CAF) is also observable at the neural level. A second main limitation was related to our "male" sample. On the basis of a functional magnetic resonance imaging study highlighting gender differences 
in regional brain activation to response inhibition (men activated the motor circuitry while women appeared to involve visual association or habit learning ${ }^{59}$ ), we chose in the present experiment to work only with male participants. However, previous studies showing tDCS-induced effects on inhibition did not separate males and females, ${ }^{21-23}$ so we can deliberate about whether similar results would have been obtained with female participants. Further studies should verify the generalizability of our data to the female population.

To conclude, tDCS seems to be of particular interest in terms of its applications in clinical neuropsychiatry and cognitive neurorehabilitation. However, current data reported controversial results and quite small effect sizes, as important factors such as best electrode localization and number of sessions, should still be better defined. By allowing a dynamic monitoring of inhibitory responses, CAF could be of the greatest relevance for researchers interested in long-term boosting of inhibitory functions.

\section{Authors' Note}

The funders had no role in study design, data collection and analysis, decision to publish, or preparation of the manuscript.

\section{Author Contributions}

$\mathrm{SC}$ contributed to conception and design; contributed to acquisition, analysis, and interpretation; drafted manuscript; critically revised manuscript; gave final approval; agrees to be accountable for all aspects of work ensuring integrity and accuracy. ES contributed to conception and design; contributed to acquisition, analysis, and interpretation; critically revised manuscript; gave final approval. MAV contributed to conception and design; contributed to acquisition; critically revised manuscript; gave final approval. CB contributed to conception and design; critically revised manuscript; gave final approval. CK critically revised manuscript; gave final approval. PV critically revised manuscript; gave final approval. BB contributed to analysis and interpretation; drafted manuscript; critically revised manuscript; gave final approval.

\section{Declaration of Conflicting Interests}

The author(s) declared no potential conflicts of interest with respect to the research, authorship, and/or publication of this article.

\section{Funding}

The author(s) disclosed receipt of the following financial support for the research, authorship, and/or publication of this article: Salvatore Campanella is Senior Research Associate with the Belgian Fund of Scientific Research (F.R.S.-F.N.R.S.). Elisa Schröder is a Research Fellow at the Fond pour la Recherche en Sciences Humaines (FRESH) from the Belgian Fund for Scientific Research (FRS-FNRS, Belgium). This work was also supported by the Ghent University Multidisciplinary Research Partnership "The integrative neuroscience of behavioral control".

\section{References}

1. Logan G, Cowan W. On the ability to inhibit thought and action: a theory of an act of control. Psychol Rev. 1984;91:295-327.

2. Dambacher F, Sack AT, Lobbestael J, Arntz A, Brugman S, Schuhmann T. The role of right prefrontal and medial cortex in response inhibition: interfering with action restraint and action cancellation using transcranial magnetic brain stimulation. $J$ Cogn Neurosci. 2014;26:1775-1784.

3. Dambacher F, Sack AT, Lobbestael J, Arntz A, Brugman S, Schuhmann T. A network approach to response inhibition: dissociating functional connectivity of neural components involved in action restraint and action cancellation. Eur $J$ Neurosci. 2014;39:821-831.

4. Petit G, Kornreich C, Noël X, Verbanck P, Campanella S. Alcohol-related context modulates performance of social drinkers in a visual Go/No-go task: a preliminary assessment of eventrelated potentials. PloS One. 2012;7:e37466.

5. Dalley JW, Everitt BJ, Robbins TW. Impulsivity, compulsivity, and top down cognitive control. Neuron. 2011;69:680-694.

6. Allom V, Mullan B, Hagger M. Does inhibitory control training improve health behaviour? A meta-analysis. Health Psychol Rev. 2016;10:168-186.

7. Beeli G, Casutt G, Baumgartner T, Jancke L. Modulating presence and impulsiveness by external stimulation of the brain. Behav Brain Funct. 2008;4:33.

8. Hsu TY, Tseng LY, Yu JX, et al. Modulating inhibitory control with direct current stimulation of the superior medial frontal cortex. Neuroimage. 2011;56:2249-2257.

9. Batista EK, Klauss J, Fregni F, Nitsche MA, Nakamura-Palacios E. A randomized placebo-controlled trial of targeted prefrontal cortex modulation with bilateral tDCS in patients with crack-cocaine dependence. Int J Neuropsychopharmacol. 2015;18:pyv066.

10. Boggio PS, Zaghi S, Villani AB, Fecteau S, Pascual-Leone A, Fregni F. Modulation of risk-taking in marijuana users by transcranial direct current stimulation (tDCS) of the dorsolateral prefrontal cortex (DLPFC). Drug Alcohol Depend. 2010;112:220-225.

11. Sauvaget A, Trojak B, Bulteau S, et al. Transcranial direct current stimulation (tDCS) in behavioral and food addiction: a systematic review of efficacy, technical, and methodological issues. Front Neurosci. 2015;9:349.

12. Fecteau S, Agosta S, Hone-Blanchet A, et al. Modulation of smoking and decision-making behaviors with transcranial direct current stimulation in tobacco smokers: a preliminary study. Drug Alcohol Depend. 2014;140:78-84.

13. Boggio PS, Sultani N, Fecteau S, et al. Prefrontal cortex modulation using transcranial DC stimulation reduces alcohol craving: a double-blind, sham-controlled study. Drug Alcohol Depend. 2008;92:55-60.

14. Jansen JM, Daams JG, Koeter MW, Veltman DJ, van den Brink W, Goudriaan AE. Effects of non-invasive neurostimulation on craving: a meta-analysis. Neurosci Biobehav Rev. 2013;37(10 pt 2):2472-2480.

15. Lefaucheur JP, Antal A, Ayache SS, et al. Evidence-based guidelines on the therapeutic use of transcranial direct current stimulation (tDCS). Clin Neurophysiol. 2017;128:56-92.

16. Spagnolo PA, Goldman D. Neuromodulation interventions for addictive disorders: challenges, promise, and roadmap for future research. Brain. 2017;140:1183-1203. doi:10.1093/brain/aww284.

17. Klauss J, Penido Pinheiro LC, Silva Merlo BL, et al. A randomized controlled trial of targeted prefrontal cortex modulation with tDCS in patients with alcohol dependence. Int $J$ Neuropsychopharmacol. 2014;17:1793-1803.

18. da Silva MC, Conti CL, Klauss J, et al. Behavioral effects of transcranial direct current stimulation (tDCS) induced dorsolateral prefrontal cortex plasticity in alcohol dependence. $J$ Physiol Paris. 2013;107:493-502. 
19. Kekic M, Boysen E, Campbell IC, Schmidt U. A systematic review of the clinical efficacy of transcranial direct current stimulation (tDCS) in psychiatric disorders. J Psychiatr Res. 2016;74:70-86.

20. Reinhart RM, Cosman JD, Fukuda K, Woodman GF. Using transcranial direct-current stimulation (tDCS) to understand cognitive processing. Atten Percept Psychophys. 2017;79:3-23.

21. Jacobson L, Javitt DC, Lavidor M. Activation of inhibition: diminishing impulsive behavior by direct current stimulation over the inferior frontal gyrus. J Cogn Neurosci. 2011;23:3380-3387.

22. Stramaccia DF, Penolazzi B, Sartori G, Braga M, Mondini S, Galfano G. Assessing the effects of tDCS over a delayed response inhibition task by targeting the right inferior frontal gyrus and right dorsolateral prefrontal cortex. Exp Brain Res. 2015;233:2283-2290.

23. Cunillera T, Fuentemilla L, Brignani D, Cucurell D, Miniussi C. A simultaneous modulation of reactive and proactive inhibition processes by anodal tDCS on the right inferior frontal cortex. PLoS One. 2014;9:e113537.

24. Jacobson L, Ezra A, Berger U, Lavidor M. Modulating oscillatory brain activity correlates of behavioral inhibition using transcranial direct current stimulation. Clin Neurophysiol. 2012;123:979-984.

25. Kelley N, Hortensius R, Harmon-Jones E. When anger leads to rumination: Induction of relative right frontal cortical activity with transcranial direct current stimulation increases angerrelated rumination. Psychol Sci. 2013;24:475-481.

26. Vartanian O, Kwantes PJ, Mandel DR, et al. Right inferior frontal gyrus activation as a neural marker of successful lying. Front Hum Neurosci. 2013;7:616.

27. Schlaug G, Marchina S, Wan CY. The use of non-invasive brain stimulation techniques to facilitate recovery from post-stroke aphasia. Neuropsychol Rev. 2011;21:288-301.

28. Braver T. The variable nature of cognitive control: a dual mechanisms framework. Trends Cogn Sci. 2012;16:106-113.

29. Cai Y, Li S, Liu J, et al. The role of the frontal and parietal cortex in proactive and reactive inhibitory control: a transcranial direct current stimulation study. $J$ Cogn Neurosci. 2016;28:177-186.

30. Cunillera T, Brignani D, Cucurell D, Fuentemilla L, Miniussi C. The right inferior frontal cortex in response inhibition: a tDCSERP co-registration study. Neuroimage. 2016;140:66-75.

31. den Uyl TE, Gladwin TE, Wiers RW. Transcranial direct current stimulation, implicit alcohol associations and craving. Biol Psychol. 2015;105:37-42.

32. Dambacher F, Schuhmann T, Lobbestael J, Arntz A, Brugman S, Sack AT. No effects of bilateral tDCS over inferior frontal gyrus on response inhibition and aggression. PloS One. 2015;10:e0132170.

33. Campanella S, Schroder E, Monnart A, et al. Transcranial direct current stimulation over the right frontal inferior cortex decreases neural activity needed to achieve inhibition: a double-blind ERP study in a male population. Clin EEG Neurosci. 2017;48:176-188.

34. Smith J, Jamadar S, Provost AL, Michie P. Motor and non-motor inhibition in the Go/NoGo task: an ERP and fMRI study. Int $J$ Psychophysiol. 2013;87:244-253.

35. Pfefferbaum A, Ford JM, Weller BJ, Kopell BS. ERPs to response production and inhibition. Electroencephalogr Clin Neurophysiol. 1985;60:423-434.

36. Yang B, Yang S, Zhao L, Yin L, Liu X, An S. Event-related potentials in a Go/NoGo task of abnormal response inhibition in heroin addicts. Sci China C Life Sci. 2009;52:780-788.
37. Jonker TR, Seli P, Cheyne JA, Smilek D. Performance reactivity in a continuous-performance task: Implications for understanding post-error behavior. Conscious Cogn. 2013;22:1468-1476.

38. van den Wildenberg WP, Wylie SA, Forstmann BU, Burle B, Hasbroucq T, Ridderinkhof KR. To head or to heed? Beyond the surface of selective action inhibition: a review. Front Hum Neurosci. 2010;4:222. doi:10.3389/fnhum.2010.00222.

39. Richard Ridderinkhof K, Forstmann BU, Wylie S, Burle B, van den, Wildenberg WPM. Neurocognitive mechanisms of action control: resisting the call of the Sirens. Wiley Interdiscip Rev Cogn Sci. 2011;2:174-192.

40. Band G, Ridderinkhof KR, van der Molen MW. Speed-accuracy modulation in case of conflict: the roles of activation and inhibition. Psychol Res. 2003;67:266-279.

41. Wylie S, van den Wildenberg WP, Ridderinkhof KR, et al. The effect of speed-accuracy strategy on response interference control in Parkinson's disease. Neuropsychologia. 2009;47:1844-1853.

42. can Vliet I, de Beurs E. The MINI-International Neuropsychiatric Interview. A brief structured diagnostic psychiatric interview for DSM-IV en ICD-10 psychiatric disorders [in Dutch]. Tijdschr Psychiatr. 2007;49:393-397.

43. Schippers GM, Broekman TG.De AUDIT. Nederlandse vertaling van de alcohol use disorders identification test. http://www. mateinfo.nl/audit/audit-nl.pdf. Accessed April 28, 2018.

44. Lijffijt M, Barratt ES. Persoonlijke evaluatie: BIS-11. http:// www.impulsivity.org/measurement/BIS-11Dutch.pdf. Accessed April 28, 2018.

45. Van der Ploeg H. Validity of the Zelf-Beoordelings-Vragenlijst (A Dutch version of the Spielberger State-Trait Anxiety Inventory). Nederlands Tijdschr Psychol Grensgebieden. 1980;35:243-249.

46. van der Does AJW. BDI-II-NL. Handleiding. De Nederlandse versie van de beck depression inventory. San Antonio, TX/Lisse, NL: The Psychological Corporation/Swets Test Publishers; 2002.

47. Ratcliff R. Group reaction time distributions and an analysis of distribution statistics. Psychol Bull. 1979;86:446-461.

48. Cousineau D. Confidence intervals in within-subject designs: a simpler solution to Loftus and Masson's method. Tutorials Quant Methods Psychol. 2005;1:42-45.

49. Morey RD. Confidence intervals from normalized data: a correction to Cousineau (2005). Tutorials Quant Methods Psychol. 2008;4:61-64.

50. Simmonds DJ, Pekar JJ, Mostofsky SH. Meta-analysis of Go/ No-go tasks demonstrating that fMRI activation associated with response inhibition is task-dependent. Neuropsychologia. 2008;46:224-232.

51. Thompson RA. Emotion regulation: a theme in search of a definition. Monogr Soc Res Child Dev. 1994;59:25-52.

52. Cole PM, Martin SE, Dennis TA. Emotion regulation as a scientific construct: methodological challenges and directions for child development research. Child Dev. 2004;75:317-333.

53. Niemi P, Näätänen R. Foreperiod and simple reaction time. Psychol Bull 1981;89:133-162.

54. Ridderinkhof KR. Activation and suppression in conflict tasks: empirical clarification through distributional analyses. In Prinz W, Hommel B eds. Common Mechanisms in Perception and Action. Attention \& Performance. Vol 19. Oxford, England: Oxford University; 2002:494-519.

55. Kornblum S, Hasbroucq T, Osman A. Dimensional overlap: cognitive basis for stimulus-response compatibility - a model and taxonomy. Psychol Rev. 1990;97:253-270. 
56. Aron AR, Robbins TW, Poldrack RA. Inhibition and the right inferior frontal cortex. Trends Cogn Sci. 2004;18:170-177.

57. Spieser L, van den Wildenberg W, Hasbroucq T, Ridderinkhof $\mathrm{KR}$, Burle B. Controlling your impulses: electrical stimulation of the human supplementary motor complex prevents impulsive errors. J Neurosci. 2015;35:3010-3015.
58. Shiozawa P, Fregni F, Benseñor IM, et al. Transcranial direct current stimulation for major depression: an updated systematic review and meta-analysis. Int J Neuropsychopharmacol. 2014;17:1443-1452.

59. Ray Li C, Huang C, Constable RT, Sinha R. Gender differences in the neural correlates of response inhibition during a stop signal task. Neuroimage. 2006;32:1918-1929. 